R.D. Grygoryan, O.I.Yurchak, A.G. Degoda, T.V. Lyudovyk

\title{
SPECIALIZED SOFTWARE FOR SIMULATING THE MULTIPLE CONTROL AND MODULATIONS OF HUMAN HEMODYNAMICS
}

\begin{abstract}
Special PC-based software simulating quantitative models of mechanisms that provide the overall control of human circulation is created. The autonomous software essentially expands the range of tasks concerning the modeling of cardiovascular physiology, in particular, of mechanisms controlling cardiac function, vascular hemodynamics, and total blood volume under unstable internal/external physiochemical environments. The models are verified on data representing hemodynamic responses to certain physical tests. The user-friendly interface provides all stages of preparation and analysis of computer simulation. The PCbased simulator can also be used for educational purposes.

Key words: physiology, cardiovascular system, acute and long-term control, model, simulator.
\end{abstract}

\section{Introduction}

Modern technologies providing the quantitative mathematical (computer) modeling of human physiological systems and the creation of specialized simulators (SS) [1-3] meet three independent problems. The first one is how to choose the right physiological concept to be modeled. The second one is how to apply adequate mathematics. The last problem concerns programming technologies (PT). Especially, a user-friendly interface (UI) development finally plays the most decisive role in the "fate" (usability) of SS.

So, before starting the code creation, a big hidden pre-work has been done. The chosen biological concept is the main stage result. Usually, programmers, involved in complex projects concerning biology are not aware of serious problems arising on this stage of the work. The matter is that in contrast with the commonly modeled objects (e.g. in industrial technologies), the model of every physiological system reflects both the level of science and the fact of simultaneous existence of competitive conceptual views of the same biological object or process. So, the team leader should have a high level of professional knowledge and proper biological background for choosing the most matching physiological concept.

The cardiovascular system (CVS) is one of the most often modeled human physiological systems [1-6,]. Purposes of the modeling determine both the complexity of CVS model and the modeling method. The lumped-parametric approach is the mostly used method for CVS's modeling [1-3]. If the number of vascular compartments in the model is properly large, this method provides the accuracy of regional circulation simulation. However, the larger this number is the smaller the numerical simulation step is and the larger calculations are. Therefore, models created for numerical simulations on PC have to match the capabilities of current PCs. On the other hand, every model has to be in some manner identified and validated for control situations. The detailed presentation of the vascular net meets with the problem that data required for the model validation are often absent. Both these opposite demands result in a workable compromise model. Another big problem of CVS's modeling concerns the endogenic mechanisms. The matter is that not all the concerned mechanisms are circulation controllers. Some of the feedback mechanisms really control CVS's parameters while others only modulate them. In order to investigate the physiological roles of mechanisms directly or indirectly modulating the current states of the heart and vasculature, the modeler needs a proper physiological concept concerning goals of so-called control mechanisms. The biggest problem is that there is yet no commonly accepted concept. Therefore, the concept accepted by the modeler usually reflects his subjective motives.

This publication aims to illustrate both internal connections of the triad of problems and the ways of these problems 
solution during the special software development. Commonly accepted physiological concepts concerning arterial pressure neural-hormonal control are modified by new ideas proposed in $[9,10]$.

\section{A short description of the basic model}

SS is based on a complex quantitative mathematical model which presents the human CVS as an open system interacting with a certain number of associated physiological systems (APS). Within the framework of traditional physiology some of these APS are known as circulation controllers. They could influence the total blood volume dynamics and current values of CVS's parameters.

The core model and models of certain APS are described in [5-9] while models of additional APS are described in this paper.

It is worth to stress here that the core model of hemodynamics contains of two submodels separately describing pump functions of the right and left ventricles respectively. The third sub-model describes hemodynamics in a vascular net of 21 lumped-parametric arterial and venous compartments. Each vascular section possesses its own constant initial characteristics (rigidity, unstressed volume, and resistance between neighboring lumped-parametric vessel sections), [5]. In addition, the core model assumes the total blood volume is constant. But in the complex model, the total blood volume is under influences of certain endogenic mechanisms. Namely, each of the so-called control mechanisms is able to alter the value of at last one of the cardiovascular parameters included in the core model.

Both ventricles are modeled similarly as a flow generator: it connects the mean output flow of ventricles with their constant parameters and variable mean input pressure [5]. The transforming function of the ventricle takes into account its structurally determined constants, as well as its inotropic state. The stable heart rate on its autonomous level transforms ventricles' output to a heart output in $\mathrm{ml} /$ sec. So, the heart model does not imitate cardiac pulsatile behavior but is a model relative to mean values of pressures and flows.

Figure 1 represents structure of the basic model. There are eight mechanisms each of them specifically influences (controls) parameters of a core model representing CVS.

In our complex model, main simulations do cover long time periods (tens of minutes, hours, and days), thus the pulsatile model of a heart pump function (HPF) [4] is substituted by the model describing HPF as a generator of mean blood flow.

In fact, the HPF is substituted by two similar models describing quasi-static relationships of ventricular input - output variables depending on much more inertial immanent characteristics of right or left ventricle. Input variables are central venous $\left(P_{v}(t)\right)$, and lung vein $\left(P_{l v}(t)\right)$ pressures. Ventricles' end-diastolic capacity $\left(C_{r}(t), C_{l}(t)\right)$, output valve resistances $\left.\left(R_{o v r}(t), R_{o v l}(t)\right)\right)$, and inotropic coefficients $\left(k_{r}(t), k_{l}(t)\right)$ are connected with their input pressures $\left(P_{v}(t), P_{l v}(t)\right)$ and output flows $\left(Q_{r}(t) Q_{l}(t)\right)$ by identical functional relationships of:

$$
\begin{aligned}
& \left.Q_{r}(t)=f_{r}\left(P_{v}(t), C_{r}(t), R_{o v r}(t)\right), k_{r}(t)\right), \\
& \left.Q_{l}(t)=f_{l}\left(P_{l v}(t), C_{l}(t), R_{o v l}(t)\right), k_{l}(t)\right) .
\end{aligned}
$$

The principle is that the core model of CVS has six independent channels flows throw which can alter the total blood volume.

In this version of the complex model, only for some of these channels the resistance is internally connected with activities of associated physiological control mechanisms (PCM). In the remaining hemodynamic channels, values of hydrodynamic resistances can be directly set through UI.

Models of seven independently acting PCM have been created:

- Mechanoreceptor reflexes initiated by receptors located in aortic arc, carotid sinuses, lung artery, and brain arteries of the circle of Willis;

- Peripheral chemoreceptor reflexes activated under alterations of arterial blood $\mathrm{PaCO} 2, \mathrm{PaO} 2$, and $\mathrm{pH}$;

- Central and local renin-angiotensin-aldosterone mechanism: it is a negative feedback mechanism activated under low local flows in kidneys or other organs. Vascular tones are the main effectors.

- Antidiuretic hormone mechanism: this model reciprocally connects the increased concentrations of aldosterone with decrease of the rate of diuresis. 


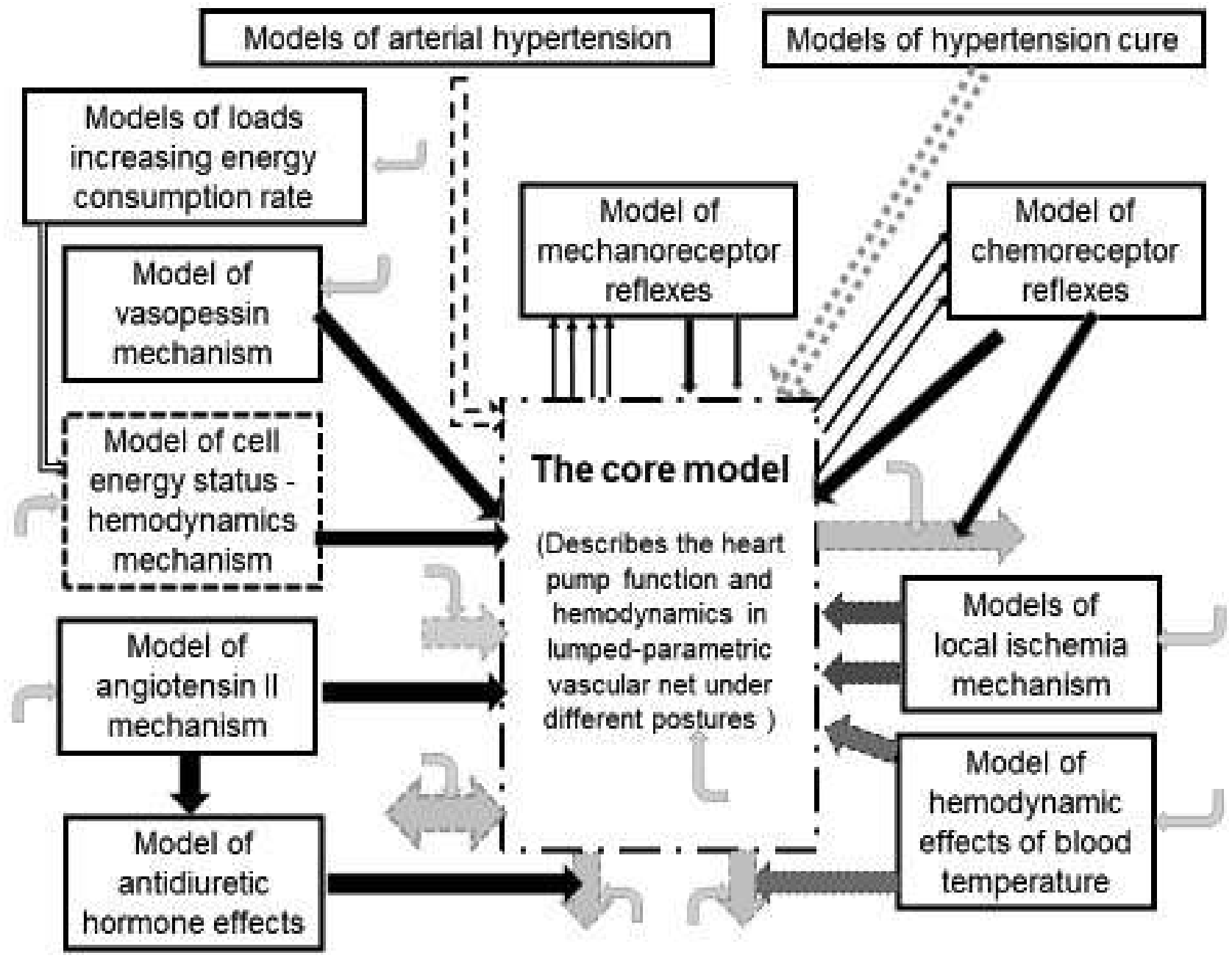

Fig.1. Structure of complex model necessary and sufficient for simulation of mechanisms controlling or modulating human hemodynamics under external / internal influences.

- Mechanisms activated under energy deficiency (EDM).

- Regional ischemia.

- Models of blood temperature influence on hemodynamics.

Special attention should be drawn to the model for the first time describing EDM. In human cells, as in every unicellular aerobic organism, there are two ways for a synthesis of energy (molecules of ATP). One way providing less than $5 \%$ of ATP molecules is their anaerobe synthesis using glucose (glycolysis). The byproduct of this synthesis is pyruvate which enters into mitochondria and its oxygenation provides about $95 \%$ of ATP. So, in ATP production oxygen, along with carbohydrates, plays the main role. Under low or moderate deficiency of ATP, oxygenassociated regulator effects can be modeled through chemoreceptor reflexes [6]. Their effects on lung ventilation and hemodynam- ics are well-known. However, under acute severe energy deficiency, the chemoreflector increases of arterial pressure and blood flows are not sufficient for overcoming the inhibited metabolism in big groups of cells. There are additional enhancers of ATP synthesis.

The mechanism of AMP-activated protein kinase is a way for compensating the glucose lack [9]. But more important is the glucagon-glucose mechanism activating liver glycogen back-transformation to blood glucose. In parallel with this, under glucose lack a peptide (glucagon), entering into blood, activates both the chronotropic and the inotropic states of the heart [9]. So, EDM has an additional opportunity to modulate the circulation. The last effects have been modeled for the first time as follows.

$$
T_{G} \frac{d G(t)}{d t}=G(t)+I(t)-\varpi_{1} \cdot W(t)
$$




$$
T_{g} \frac{d g(t)}{d t}=\left\{\begin{array}{c}
\varpi_{2} \cdot\left(G(t)-G_{c}\right)-g(t)-g_{u}, \quad G(t) \geq G_{c} \\
\varpi_{3} \cdot\left(G_{c}-G(t)\right)-g(t), \quad G_{c} \geq G(t)
\end{array}\right.
$$

In these equations, $G(t)$ and $G_{c}$ represent current and critical blood glucose concentrations, $W(t)$ is the total load, $g(t)$ is the concentration of blood glucagon, $\varpi_{1}, \varpi_{2}$, and $\varpi_{3}$ are approximation constants, $T_{G}$ and $T_{g}$ characterize inertia of glucose-glucagon transformation mechanism.

$$
\begin{gathered}
\Delta k_{g}(t)=\varpi_{4} \cdot\left(q_{C}(t)-q_{C}(0)\right) ; \\
k(t)=k(0)+\Delta k_{g}(t) ; \\
\Delta F_{g}(t)=\varpi_{5} \cdot q(t) ; \\
R_{C}(t)=R_{C}(0) \cdot\left(1-\varpi_{6} \cdot q_{C}(0) / q_{C}(t)\right.
\end{gathered}
$$

Here again $\varpi_{4}, \varpi_{5}$ and $\varpi_{6}$ are approximation constants, $\Delta k_{g}(t)$ is the glucagon-caused increase of the heart inotropic state $k(t)$ from its initial value of $k(0), q_{C}(0) \quad q_{C}(t)$ represent initial (normal) and current values of coronary blood flows, $R_{C}(0) R_{C}(t)$-appropriate resistances of coronary arteries. $\Delta F_{g}(t)$ is the glucagon-influenced increase of the heart rate.

Inotropic states of left $\left(k_{l}(t)\right)$ and right $\left(k_{r}(t)\right)$ ventricles assumed to be the same: $k_{l}(t)=k_{r}(t)=k(t)$

Each regulator has its part in summary shifts of $F(t), k_{r}(t)$ and $k_{l}(t)$, as well as in shifts of compartmental values of $D_{m}(t)$ and $U_{m}(t)$, therefore the complex model is capable to simulate the main endogenic modulations of both central and regional circulation.

$$
\begin{gathered}
F(t)=F_{a}+\sum_{j} \Delta F_{j}(t) ; \\
D_{m}(t)=D_{m}(0)+\sum_{m} \Delta D_{m}(t) ; \\
U_{m}(t)=U_{m}(0)-\sum_{m} \Delta U_{m}(t) ;
\end{gathered}
$$

The core model was created in assumption that CVS's characteristics including the total blood volume $V_{T}(t)$ are constant. Above it was shown that both heart and vascular characteristics are under regulators influences. It is known that under long-time observations, the value of $V_{T}(t)$ is also altered. Alterations depend on changes of the balance between liquid inflows into CVS and outflows from CVS. So, CVS is an open system interacting with multiple organs (kidneys, skin, lungs), intercellular space, as well as with the digestive system. Most of these participants are under specific regulators that have not been yet definitely described in the complex model. At the same time, empty arrows in Fig.1 depict the fact that the UI provides the user with direct alterations of certain arbitrarily chosen liquid flows ( $\Delta q_{n}$ ). Thanks to such manipulations, the physiologist will be able to watch hemodynamic effects induced by each such alteration.

$$
\frac{d V_{T}(t)}{d t}=\sum_{n} \Delta q_{n}(t)
$$

In Fig.1, arrows like indicate that the corresponding model also has direct inputs provided by UI. Combinations of multiple arrows illustrate that values of associate input or output flow can be directly modulated by means of UI. These temporarily implemented additional options have been used to provide first (rough) assessments concerning the likely contribution of real mechanisms modulating these flows in the intact organism.

\section{Simulation algorithms}

A single simulation algorithm (SA) depends on: 1) actual configuration of physiological models (ACPM); and 2) actual group of input loads (AGIL). This can be illustrated by means of Fig. 2 which represents the general view on SA.

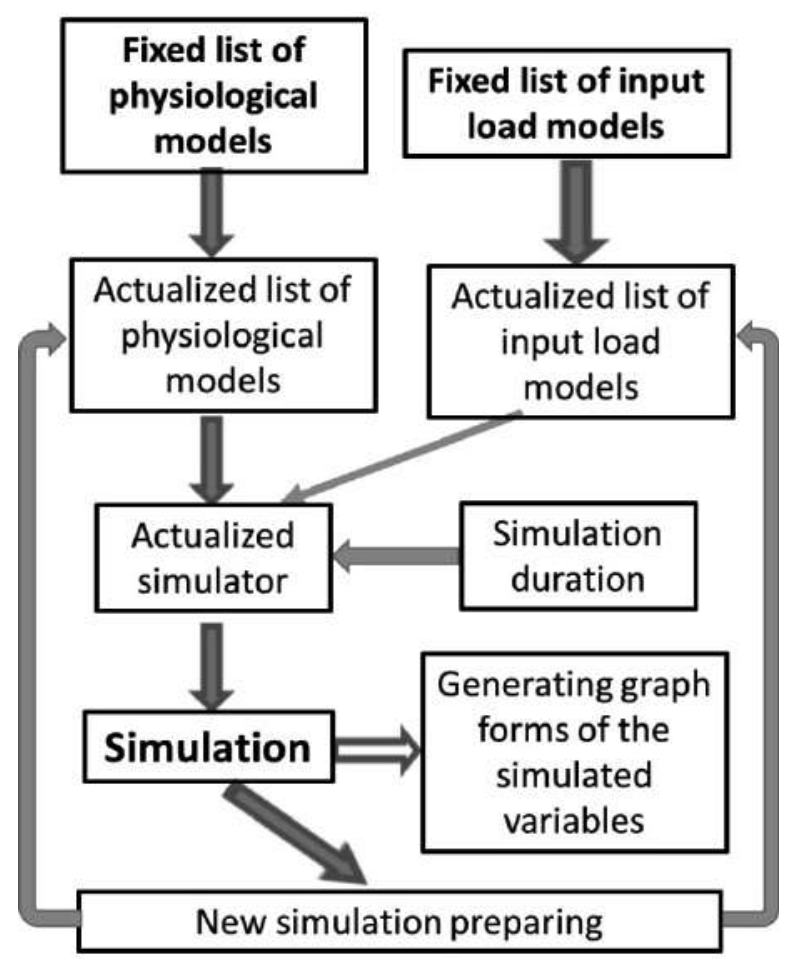

Fig.2. Simulation algorithms. 
According to this algorithm, two independent procedures have to be performed before the simulator is ready to execute calculations. As a result of the first procedure the user gets the actualized ACPM. The second procedure generates AIGL. Additionally, the user should set the simulation duration. Changing at least one value in characteristics of ACPM and/or AGIL, the user can start next simulation.

Potentially, our simulator consists of 12 independently functioning physiological models and 10 models each representing one dynamic input load. So, the number of actualized ACPM and AGIL is too large. In fact, no empirical physiologist has ever observed hemodynamic effects of entire scenarios provided by our simulator. The user will be able to run and analyze the entire spectrum of simulations, he / she will be provided by an effective user interface.

\section{Input loads}

Our models and the entire SS imitate dynamic physiological responses of a healthy person to dynamic input loads. Namely, the response depends on the absolute level and shape of the applied load. Theoretically, it is possible to create a simulator providing the construction of every arbitrary load profile. Currently, our SS provides only fixed input loads each having its specific shape. At the same time, the shape is set based on the common trapezoidal shape formally describing the input load $W(t)$ as:

$$
W(t)=\left\{\begin{array}{c}
0, \quad t<T_{b} ; t>T_{e} \\
v_{u} \cdot\left(t-T_{p 1}\right) ; \quad T_{b} \leq t \leq T_{p 1} \\
W_{\max } ; \quad T_{p 1} \leq t \leq T_{p 2} \\
W_{\max }-v_{d} \cdot\left(t-T_{p 2}\right) ; \quad T_{p 2} \leq t \leq T_{e}
\end{array}\right.
$$

where

$$
\begin{aligned}
& T_{p 1}=T_{b}+W_{\max } / v_{u} ; \\
& T_{e}=T_{p 2}+W_{\max } / v_{d} .
\end{aligned}
$$

Here $T_{b}$ is time to start the loading, $T_{p 1}$ time when the maximum load $W_{\max }$ is reached; $v_{u}$ is the load increasing velocity, $v_{d}$ is the load decreasing velocity, $T_{p 2}$ is the end time of the load plateau, $T_{e}$ is the exposure end time.

All other loads (changes of total blood volume, blood temperature, occlusion of local artery) have similar shape or its reduced versions.
Controlled linear alterations of total blood volume $(V)$, namely $( \pm \Delta V)$, are provided according to formulae:

$$
\Delta V(t)=\left\{\begin{array}{c}
V_{a b}(t) \pm v_{a l} ; \quad T_{b \Delta V}<t<T_{b \Delta V} \pm \Delta V / v_{a l} \\
V_{a b}(t) ; \quad t \leq T_{b \Delta V} ; t \geq T_{b \Delta V} \pm \Delta V
\end{array}\right.
$$

where $V_{a b}(t)$ is abdominal vein volume, $T_{b \Delta V}$ is the start time for the altering of total blood volume with the velocity $v_{a l}$.

Blood temperature $\left(T^{o}(t)\right)$ alterations $\left( \pm \Delta T^{o}\right)$ alter almost linearly the heart rate $F(t)$ and regional vascular diameters. These effects have been modeled by us. In order to offer the user an access to these mechanisms, additional formulas describing activation (deactivation) of these mechanisms are needed. In our current $\mathrm{SS}$, the incorporated formulas provide setting of numerical values of normal blood temperature $\left(T_{N}^{o}\right)$ and stable velocity of temperature's elevation $\left(+v_{T}\right)$ until the maximal $\left(T_{\max }^{o}\right)$ level is reached:

$$
\begin{aligned}
& T^{o}(t)=\left\{\begin{array}{c}
T_{\max }^{o} ; \quad T^{o}(t) \geq T_{\max }^{o} \\
T_{N}^{o}+v_{T} \cdot t ; \quad t_{b T}<t<t_{e T}
\end{array}\right. \\
& t_{e T}=\left(T_{\max }^{o}-T_{N}^{o}\right) / v_{T}
\end{aligned}
$$

By analogy, under temperature lowering with stable velocity of $\left(-v_{T}\right)$, and maximal $\left(T_{\max }^{o}\right)$ or minimal $\left(T_{\min }^{o}\right)$ levels:

$$
\begin{aligned}
& T^{o}(t)=\left\{\begin{array}{cl}
T_{\min }^{o} ; & T^{o}(t) \leq T_{\min }^{o} \\
T_{N}^{o}-v_{T} \cdot t ; & t_{b T}<t<t_{e T}
\end{array}\right. \\
& t_{e T}=\left(T_{N}^{o}-T_{\min }^{o}\right) / v_{T}
\end{aligned}
$$

The tilt test is an exclusive dynamic load connected only with the angle $(A(t))$ that a body forms with the horizontal axis. Simulations of this test are possible due to the fact that in the basic model of hemodynamics, one of determinants of blood flows is the gravitational factor:

$$
G_{i}(t)=\gamma \cdot H_{i} \cdot \sin (A(t) .
$$

Here $\gamma$ is a constant transforming measures in $\mathrm{cm}$ to $\mathrm{mm} \mathrm{Hg}$, index " $i$ " concerns each vascular compartment located at a distance of $H_{i}$ from the zero level (foots).

To imitate gradual ischemia of regional (kidney, coronary, or brain) artery, two parameters are used. One determines the magnitude of local resistance increase, the other one determines its speed. Formally, the ischemia simulation is modeled as: 


$$
R_{1}(t)=\left\{\begin{array}{c}
R_{1} 0 \cdot\left(\frac{V_{i} 0}{V_{i}(t)}\right)^{2}, t<T_{b I} \\
R_{1} 0 \cdot\left(1+\Delta_{i}(t) \cdot\left(\frac{V_{i} 0}{V_{i}(t)}\right)^{2}, \quad T_{b I}<t<T_{e I},\right. \\
R_{1}\left(T_{e I}\right), \quad t>T_{e I}
\end{array}\right.
$$$$
\text { where } \Delta_{i}(t)=\Delta_{i}^{\max } \cdot\left(t-T_{b I}\right) \text {, }
$$

Constants $\Delta_{i}^{\max }, T_{b I}$ and $T_{e I}$ will be set through UI.

\section{Requirements to user interface}

According to the stated goal, our SS is a software-modeling tool (SMT). So, SMT does give the physiologist-researcher the ability to simulate almost the entire spectrum of events and situations that have ever been proposed as cardiovascular functional tests. The most known functional tests are:

1) Exogenous dynamic alterations of total blood volume;

2) Postural tests for different tilting angles;

3) Dynamic physical aerobic loads of given profiles;

4) Alterations of blood temperature;

5) Heart myocardium ischemia;

6) Brain ischemia;

7) Kidneys ischemia;

8) Shutdown of any number of control mechanisms (including the extreme case of the uncontrolled CVS).

All items on this list excluding postural tests can be combined. So, specialized UI does easily provide the physiologist with a computer experiment (simulation) very similar to experiments provided on a natural organism. Each experiment has to be specially marked for further independent analysis. Therefore, special simulation passport is necessary. The passport contains information about the characteristics of the experiment, namely, the model configuration, parameters of tests, experiment's duration, human body position.

Certainly, numerical characteristics of models do not cover the entire diapason of the regulator mechanism or possible values of test parameters. Our SMT will adequately simulate physiological responses only within certain boundaries that were verified in special investigations. Most problems, asso- ciated with the tuning and the verification of models' constants, were mainly solved by means of special software and interface described in [11]. That software provided accesses to about 450 parameters of models. But in the current software, only the most informative physiological characteristics are explicated in graph forms. Namely, the list of these characteristics includes those variables that physiologists usually try to observe and analyze in their traditional experiments. Seven groups collecting these characteristics are designed.

The first group consists of graphs representing dynamics of mean arterial pressure, systolic and diastolic arterial pressures in the aortic arch, the mean arterial pressures in the area of carotid sinus, in brain, in kidneys, in lungs, mean venous pressures in lungs veins and in central vein. Besides, the heart rate is also included in this group.

The second group is formed of graphs showing dynamics of flows. Here are collected outputs of right and left ventricles, summary flow directed to the head and its separation to flows in hands and brain, flows into abdominal organs, kidneys, legs.

The third group represents graphs of six sectional blood volumes. Body sections are associated with cavities and lungs. Such information is important for assessing the power of the regulators in different postures of a person.

The fourth group consists of graphs representing variables related to the chemoreceptor reflex. The group connects three input variables $\left(\mathrm{pH}, \mathrm{PaCO}_{2}\right.$, and $\left.\mathrm{PaO}_{2}\right)$ with afferent impulse patterns into the brain structures that, via modulating of efferent sympathetic and parasympathetic impulse patterns, alter the lung ventilation, blood concentration of hemoglobin, as well as the state of CVS. The matter is that $\mathrm{pH}, \mathrm{PaCO}_{2}$, and $\mathrm{PaO}_{2}$ depend on the total rate of energy consumption in cells.

The fifth group represents graphs of baroreceptor activities in four arterial zones: aortic arch, carotid sinus, Willis's circle, and lungs artery.

The sixth group consists of graphs representing blood concentrations of glucose, of glucagon, of angiotensin-II, and of the antidiuretic hormone. 
The seventh group collects of graphs representing characteristics of right and left ventricles.

General view of the main window of $\mathrm{SS}$ is shown in Fig.3. Namely, this window contains main commands, necessary for both preparing and executing a simulation. In addition, the window also provides the user with capabilities to look simulation results.

\section{Simulations preparing and execution}

Every simulation is an independent computer experiment with a previously collected configuration of models. Operations needed to prepare a computer simulation, as well as its executing and results analyzing, are listed in the window located on the left side of the UI, shown in Fig.3. Information concerning details of every chosen string is indicated in the right side of the UI window.

Model configuring is a multi-step operation aimed to create the desired combination of activated regulator mechanisms, tests to be applied, and simulation duration. Additional opportunities for models activation or deactivation are provided through the windows shown in right sector of the main window. Some of these windows are pop-up windows. An example of the pop-up window designed to actualize parameters of baroreceptor reflexes is shown in Fig.4.

Simulation (when activated) will last until the exposure time is over. All simulation results are saved in the operative memory thus this parameter of PC is critical for determining the maximal simulation duration.

Our simulator supports the creation of multiple biological model versions each of which is capable of providing hemodynamics under a single or more chosen input loads. In fact, these manipulations imitate empirical methods of certain control mechanisms deactivation (activation).

Special window providing tests choice is shown in Fig.5.

Below is the window for preparing simulations under a special test of aerobic load. Pay attention that the values of $\mathrm{W}(\mathrm{t})$ are given relatively to the zero level which con-

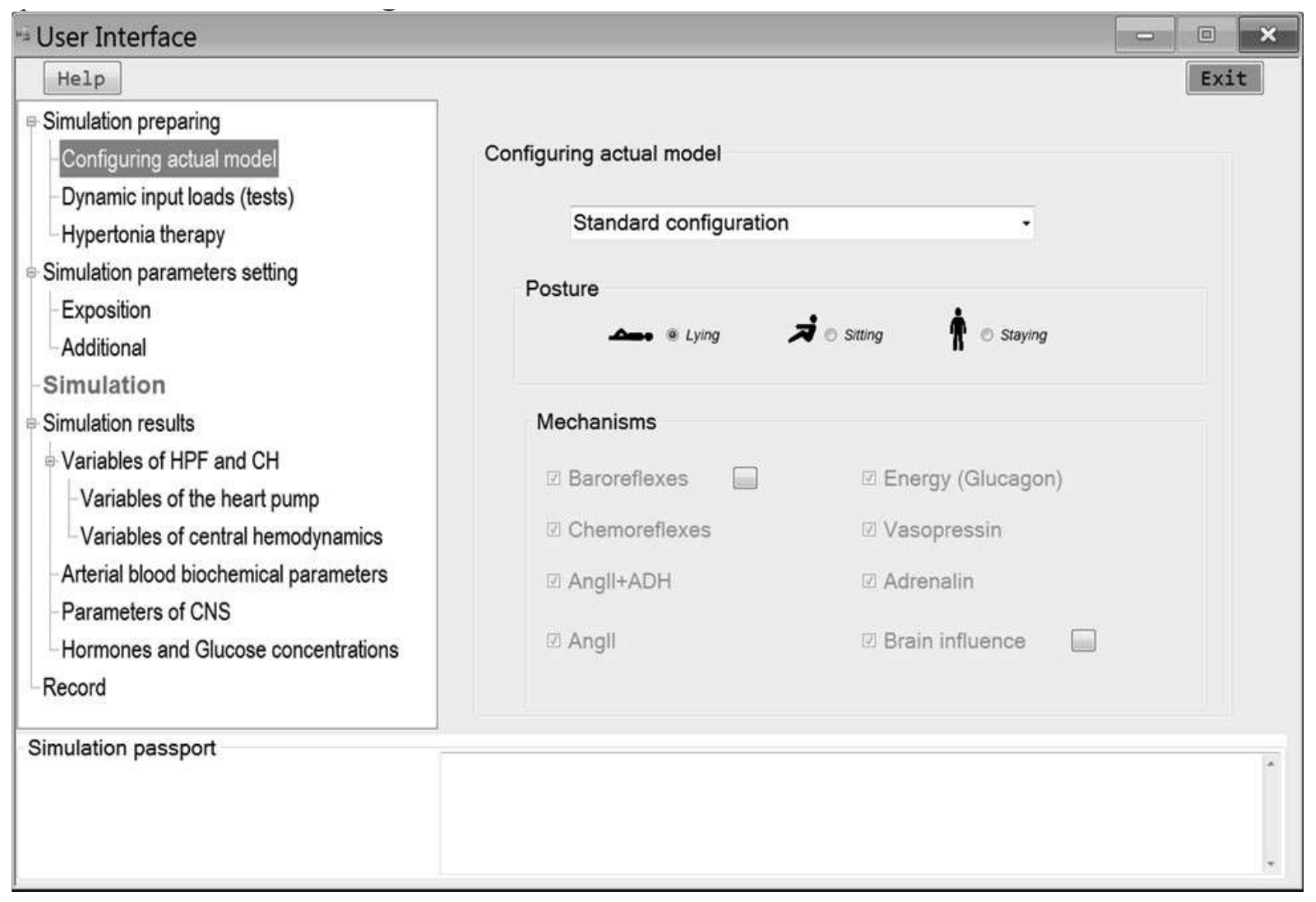

Fig.3. User interface in case of regulators' standard configuration. 


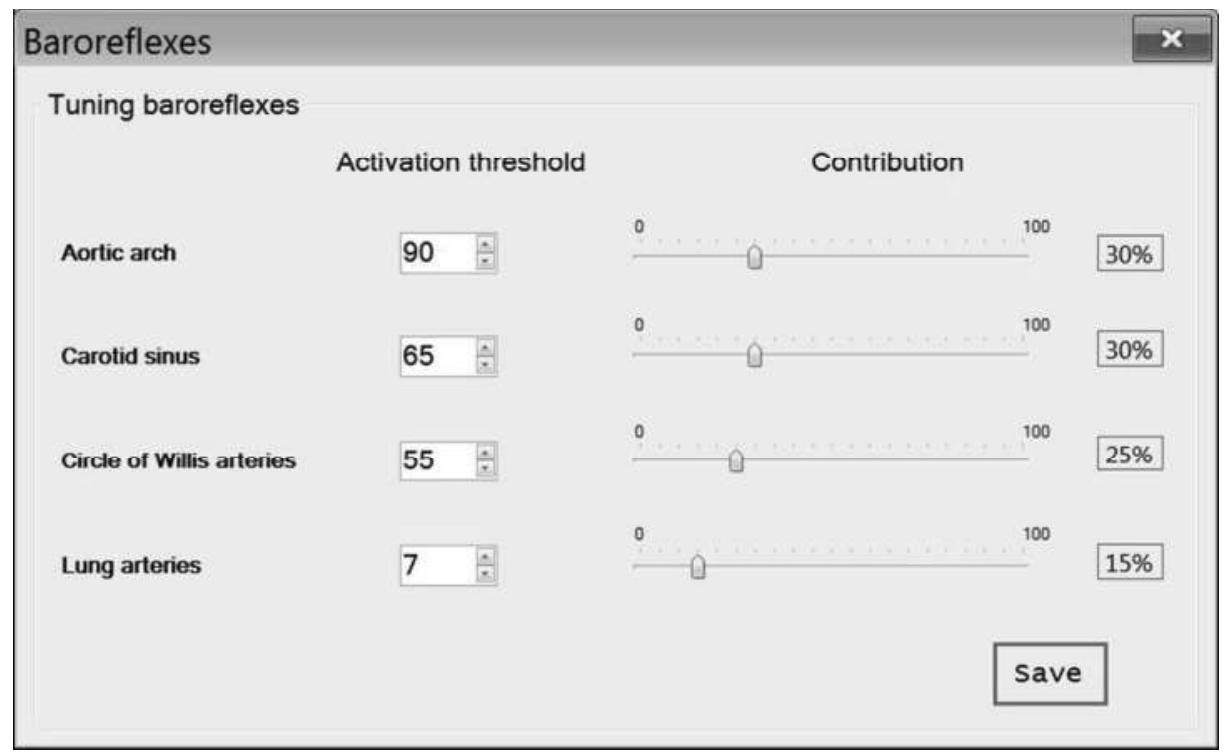

Fig.4. User interface special window for setting activation thresholds of each reflex, as well as its relative contribution to summary baroreceptor reflex.

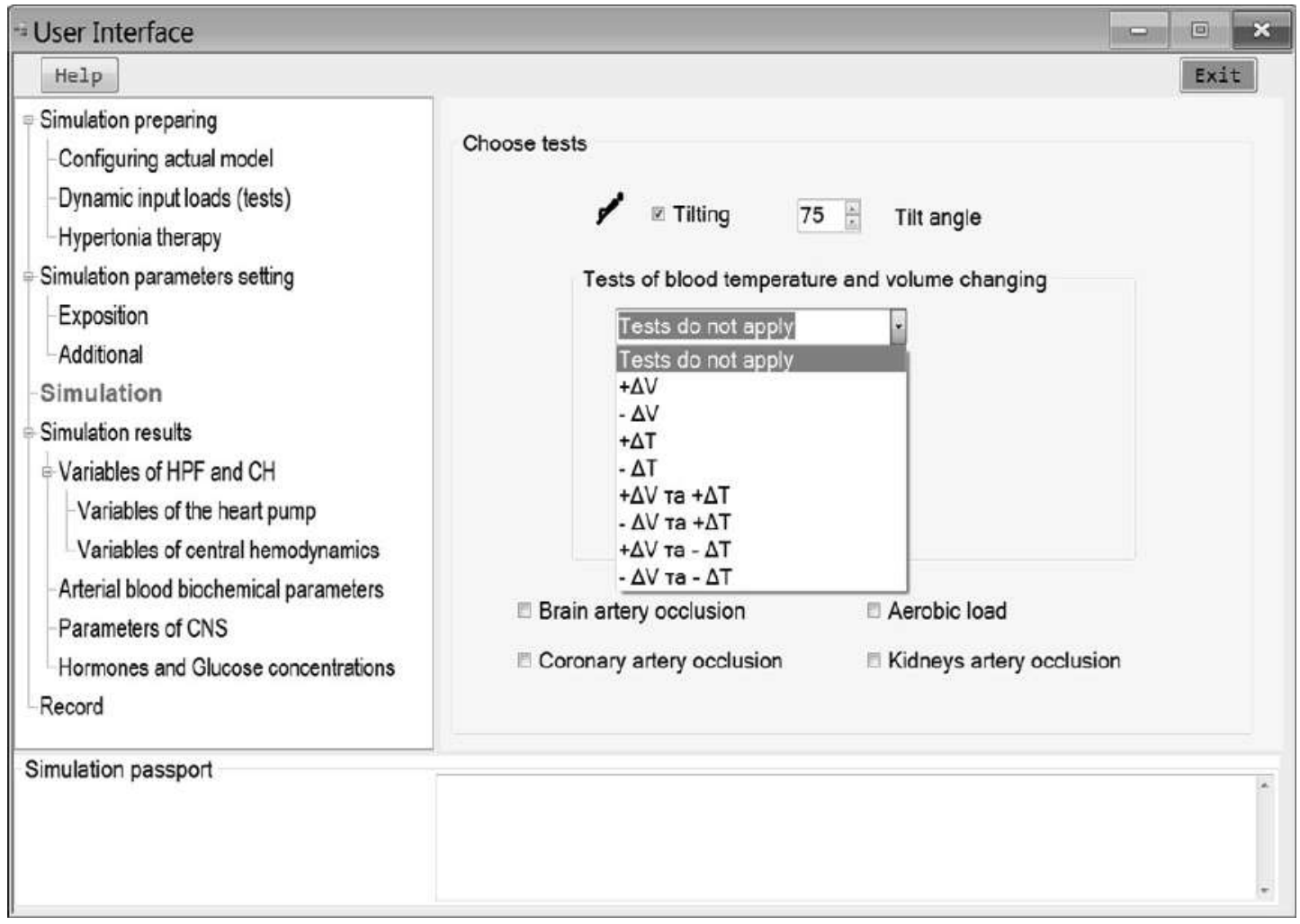

Fig.5. User interface window in case of tests performing.

ventionally represents organism's summary energy expenditures needed for providing the rest physiology in human body horizontal (clinostatic) position. The user can arbitrarily set the load starting time (TWbegin), the maximal excess load (in \% to the rest level energy expenditures), the load's increasing speed (vWup). These three parameters determine the time moment (TWPlato1) when the load reaches its Wmax. Parameter of Tstab, characterizing stable load, is also set by the user, so, the time momentum TWPlato2 for starting load decreasing with a user-defined speed of $\mathrm{vWdown}$ will determine the time momentum TWend for finishing the loading. For $\mathrm{t}>$ TWend values of $W(t)=0$. Despite this organism's responses will last until hemodynamic parameters return to their initial rest values. 


\section{Software Environment and Tools}

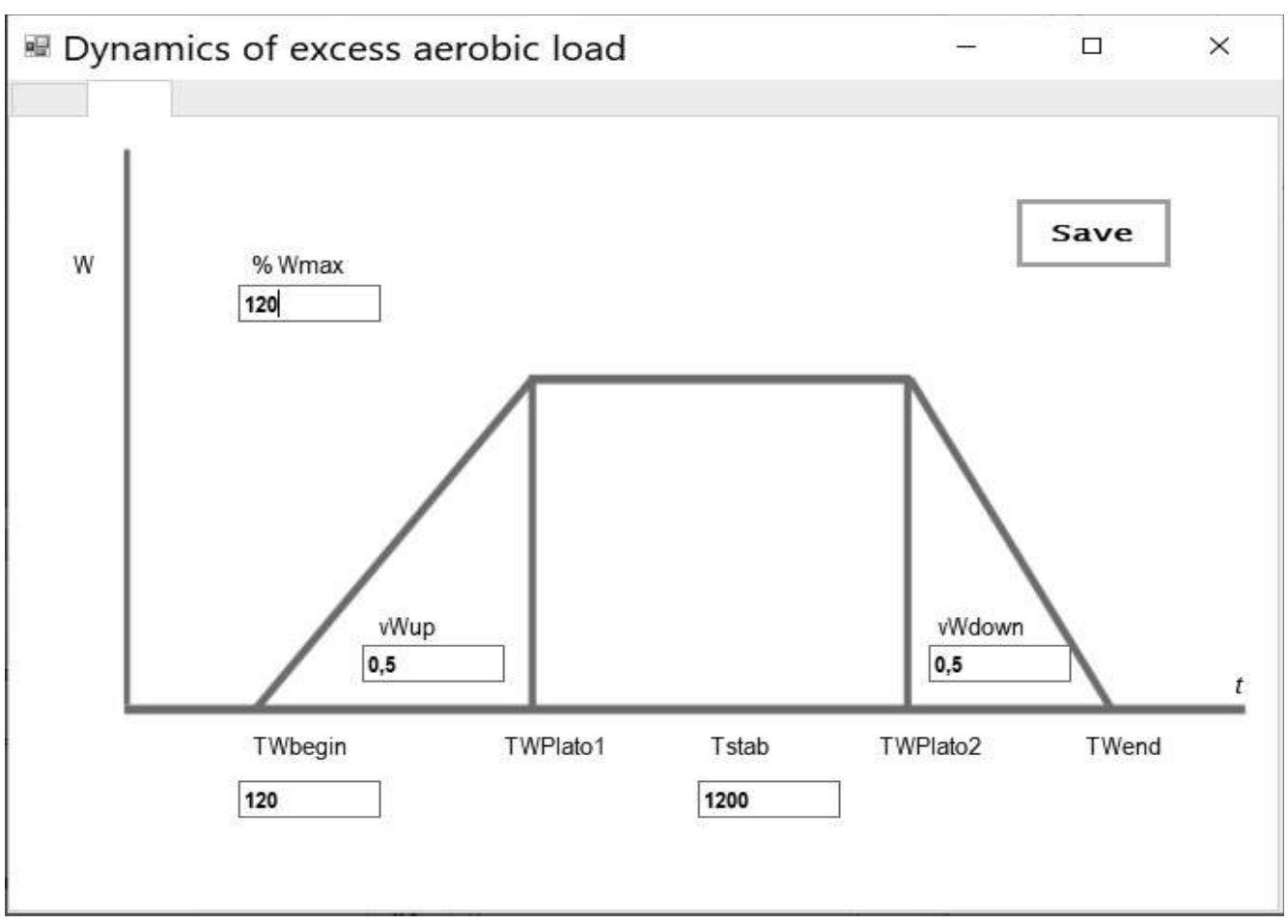

Fig.6. User interface window for setting current values of parameters of input load $W(t)$. The latter is given in a percentage of aerobic $\mathrm{W}_{\max }$. Time in sec.

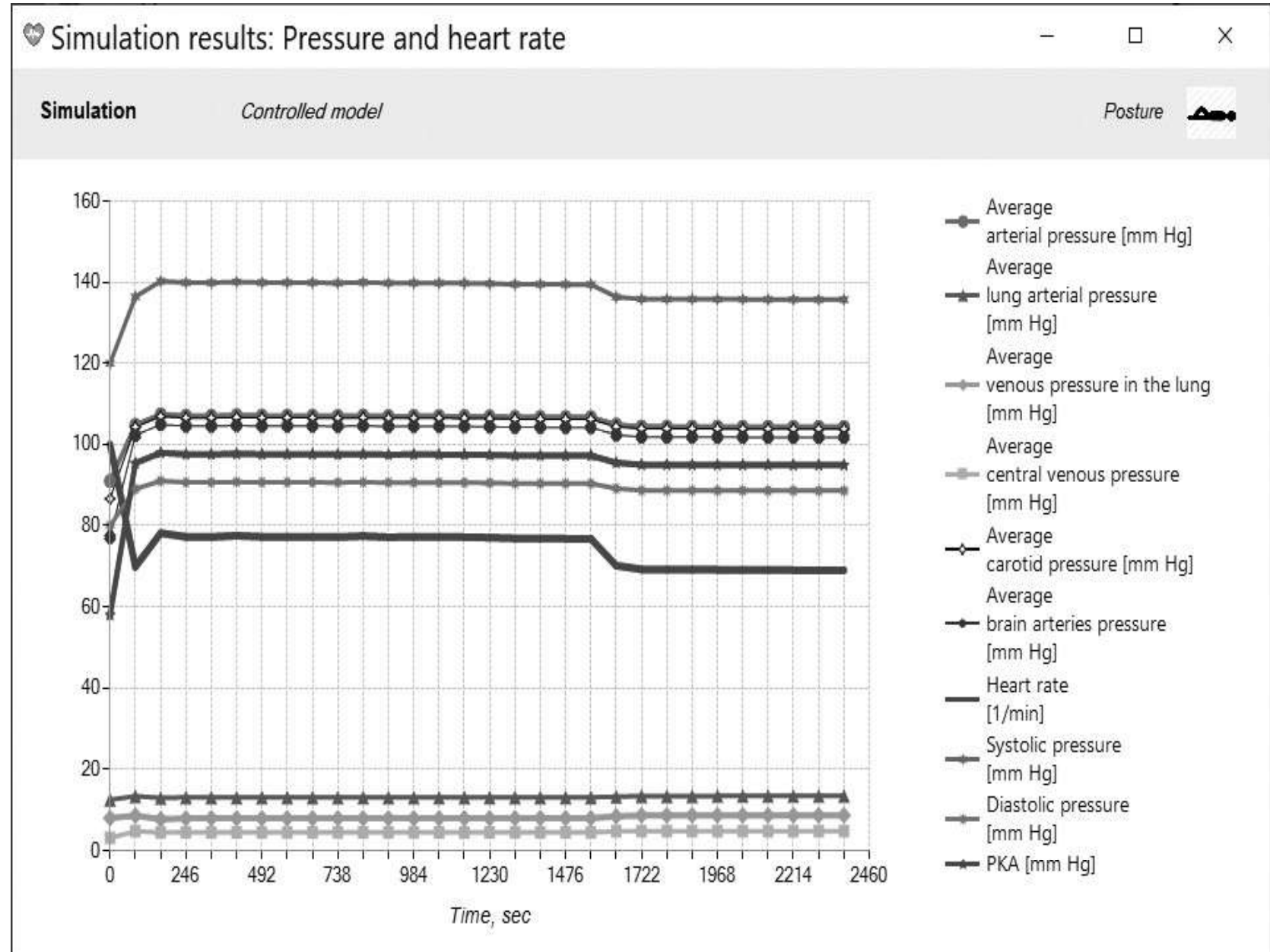

Fig.7. Main variables of central hemodynamics under aerobic load test. 
The loading profile can be saved for repetitive simulations with new actual models.

In this paper, we do not present graph results of each of the seven simulation collections. Their interface design looks similar to next pictures illustrating graphs constructed for the case presented in Fig. 6 under regulators' standard configuration and parameters of baroreceptor reflexes illustrated in Fig. 4.

It should be noted that in our current simulator, a simulation starts from data that have not been exactly tuned for the balanced hemodynamics. The exact tuning will require additional special algorithms associated with human posture. A random data set includes a certain phase of the initial transitory process that leads to balanced hemodynamics. Simulator's previous exploration has shown that the initial transitory process is almost completed at the 20-th second. So, it is much easier to use a common calculation algorithm taking into account that the simulation results are informative just for $t>20 \mathrm{sec}$. Namely, they present dynamic effects of regulators activation against hemodynamic imbalances initiated by the dynamics of W(t). Fig. 8 below shows organism's responses to this specific load.

As curves in Fig.8 show, under 100\% elevation of $\mathrm{W}(\mathrm{t})$ with an increasing speeds of $0,5 \mathrm{~W}_{\max } / \mathrm{sec}$ and decreasing speeds of $0,5 \mathrm{~W}_{\max } / \mathrm{sec}$, chemical indicators of arterial blood have significant shifts. Shapes of these shifts are similar to the shape of $\mathrm{W}(\mathrm{t})$ (see also Fig.6). Under the used elevation of $\mathrm{W}(\mathrm{t})$, decreases of $\mathrm{pO}_{2}$, increases of $\mathrm{pCO}_{2}$ are large enough to activate compensatory mechanisms of peripheral chemoreceptor reflexes. Proper increasing of lung ventilation and of the number of red blood cells are only particular compensatory effects of this reflex. Additional hemodynamic effects of chemoreceptor reflexes are shown in Fig. 7. Just W(t) returns to its zero value, all compensatory shifts are slowly returning to their initial values.

The list of human physiological characteristics used in SS is larger than those shown in Fig.7 and Fig.8. These figures only illustrate our approach to visualizing dynamic

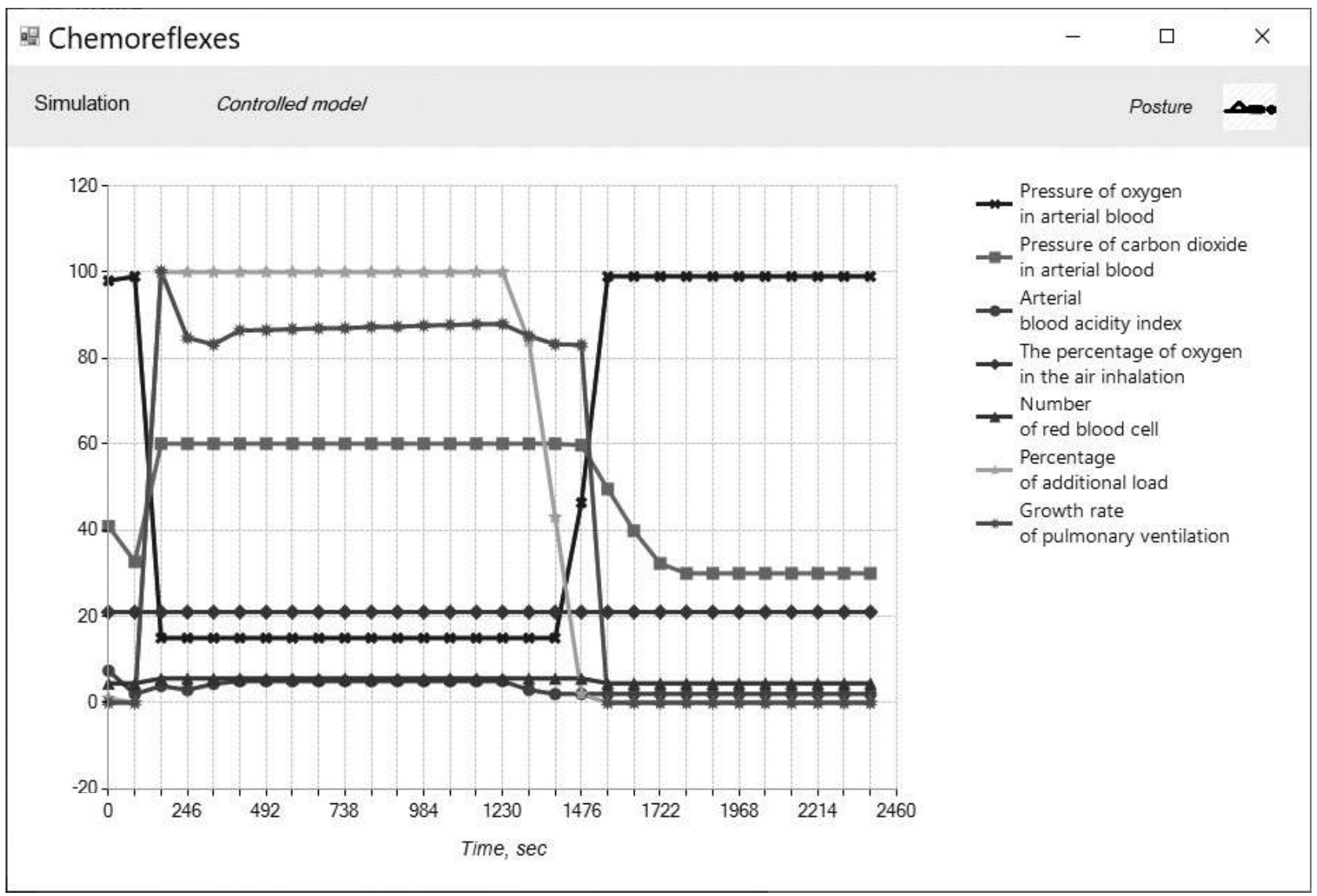

Fig.8. Illustrations of simulations for a group of variables characterizing chemoreceptor reflex. Here the aerobic load is twice as high as it is in human rest condition in clinostatics. 
physiological characteristics potentially necessary to be included by physiologists in their systematic analysis of complex relationships determining cardiovascular and associated systems' integrative responses to internal / external physical-chemical loads.

\section{Discussion}

The simulator described in the paper is autonomous software designed for IBM compatible computers. At the same time, it is the first result of the long-term fundamental investigations aimed to understand human integrative physiology by means of novel conceptual and methodological renovations. In particular, renovations concern traditional empiric research that has to be expanded with theoretical computer-based research provided by adequate quantitative simulators of physiological mechanisms and events [6-11].

As it was mentioned above, the complex model includes 11 physiological regulators each modifying certain parameters of the core model. The last one describes selfregulatory properties of a closed loop (see Fig.1). So, the complex model includes 12 regulator-associated partial models. Even in the case when the test and its parameters are chosen, the user can provide experiments for $2^{12}=5096$ combinations of activated or deactivated regulators. This number is too huge: in fact no experimenter has ever explored the full range of regulators combinations. Adding to this number also the versions that appear due to combining certain tests and modifying their parameters, one can see that the number of potential simulation scenarios becomes so big that can hardly be tested by creators of SS. At the same time, it is worth to note that the main constants of our models were tuned using specially developed assistant software [11]. These reasons give us a basis to consider our SS the first working version capable of essentially expanding the physiological research concerning mechanisms altering human hemodynamics. Further improvement of this SS needs proper feedbacks from physiologists exploring our $\mathrm{SS}$. We are sure that the exploration will accumulate additional data requiring our more thorough analysis.

\section{Conclusion}

For the first time special software (SS) capable of simulating alterations of human hemodynamics via automatic or arbitrary activations of main endogenous physiological mechanisms, is developed. $\mathrm{SS}$ is based on quantitative mathematical models representing CVS as an open system interacting with multiple associated organs and systems. Models have been tested and validated on the knowledge basis concerning physiological norm. Additionally, main hypotheses of arterial hypertension etiology can be modeled. SS provides physiologists with a novel research technology essentially widening and deepening the fundamental knowledge concerning human circulation. $\mathrm{SS}$ is also a good modern PC-based tool for simultaneously visualization of CVS's dynamic characteristics under the chosen list of input violations. The latter aspect will promote medical students to better understand non-obvious integrative human physiology and special pathologies. SS is also a good computer program to be used in educational purposes for illustrating main physiological and certain pathological regularities to medical students. We plan to expand the models and the software in order to simulate much more realistic scenarios of both normal and pathological human physiology.

\section{References}

1. Larrabide I., Blanco P.J., Urquiza S.A., Dari E.A., Ve'nere M.J., de Souza e Silva N.A., Feijo' R.A. HeMoLab - Hemodynamics Modelling Laboratory: An application for modelling the human cardiovascular system. Computers in Biology and Medicine. 2012, V. 42, P. 993-1004.

2. Fresiello L., Ferrari G., Di Molfetta A., Zieliński K., Tzallas A., Jacobs S., et al. A cardiovascular simulator tailored for training and clinical uses. J Biomed Inform 2015,57. P. 100-112.

3. Grygoryan R.D. Problem-oriented computer simulators for solving of theoretical and applied tasks of human physiology. Problems of programming. 2017, №3, P. 102-111.

4. Grygoryan R.D., Lissov P.N. A software-simulator of human cardiovascular system based on its mathematical model. Problems of program- 
ming. 2004, №4. C.100-111 (Rus).

5. Grygoryan R.D., Degoda A.G., Kharsun V.S., Dzhurinsky Y.A. A simulator of mechanisms of acute control of human hemodynamics. Problems of programming, 2019;1:90-98. (Rus.) doi.org/10.15407/pp2019.01.090.

6. Grygoryan R.D., Degoda A.G., Dzhurinsky Y.A. A simulator of mechanisms of long-term control of human hemodynamics. Problems of programming, 2019;4:111-120.(Rus). doi. org/10.15407/pp2019.04.111.

7. Grygoryan R.D., Aksenova T.V., Degoda A.G. A computer simulator of mechanisms providing energy balance in human cells. Cybernetics and computing technologies. 2017, №2 (188), P.65-73. (Rus).

8. Grygoryan R.D., Lissov P.N., Aksenova T.V., Moroz A.G. The specialized software-modeling complex "PhysiolResp". Problems of programming, 2009, 2:140-150 (Rus).

9. Grygoryan R.D. The optimal circulation: cells' contribution to arterial pressure. 2017, Nova Science, N.Y., 298 p.

10. Grygoryan R.D. The unknown aspects of arterial pressure. Znanstvena misel journal, 2019, 33:19-23.

11. Grygoryan R.D., Yurchak O.I., Degoda A.G., Lyudovyk T.V. A software technology providing tuning procedures of a quantitative model of human hemodynamics. Problems of programming, 2020;4:03-13. (Ukr). https://doi. org/10.15407/pp2020.04.003.

Received: 06.05.2021

\section{About authors:}

\section{Grygoryan Rafik}

Department chief, $\mathrm{PhD}$, D-r in biology

Publications number in Ukraine journals -147

Publications number in English journals -46.

Hirsch index -10

http://orcid.org/0000-0001-8762-733X.

Yurchak Oksana,

Leading software engineer.

Publications number in Ukraine journals - 14 .

Publications number in English journals - 0.

Hirsch index -0 .

https://orcid.org/0000-0003-3941-1555.

Degoda Anna,

Senior scientist, PhD.

Publications number in Ukraine journals - 15.

Publications number in English journals -1.

Hirsch index -3 .

http://orcid.org/0000-0001-6364-5568.

Lyudovyk Tetiana,

Senior scientist, $\mathrm{PhD}$.

Publications number in Ukraine journals - 30 .

Publications number in English journals -17.

Hirsch index -5 .

https://orcid.org/0000-0003-0209-2001.

\section{Affiliation:}

Institute of software systems of Ukraine National Academy of Sciences

03187, Kyïv,

Acad. Glushkov avenue, 40,

Phone.: 5265169.

E-mail:

rgrygoryan@gmail.com, daravatan@gmail.com, anna@silverlinecrm.com, tetyana.lyudovyk@ gmail.com 